\title{
CLINICAL SCENCE
}

\section{Isokinetic assessment of the hip muscles in patients with osteoarthritis of the knee}

\author{
Renata Alqualo Costa, Leda Magalhaes de Oliveira, Sandra Hiroko Watanabe, Anamaria Jones, \\ Jamil Natour \\ Universidade Federal de São Paulo - Disciplina de Reumatologia - São Paulo, SP, Brazil.
}

OBJECTIVES: To evaluate the difference in isokinetic strength of hip muscles between patients with knee osteoarthritis (OA) and matched healthy controls, and to establish the correlation between this isokinetic strength and pain and function in patients with knee OA.

METHODS: 25 patients with a diagnosis of unilateral knee OA, 25 patients with bilateral knee OA, and 50 matched controls were evaluated using the visual analog scale for pain, knee Lequesne index, Western Ontario and McMaster Universities questionnaire and an isokinetic test.

RESULTS: The groups were matched for age, gender and body mass index. The results of the isokinetic test revealed lower peak torque of the hip in patients with OA of the knee than in the control group for all movements studied. Strong correlations were found between the peak torque, visual analog scale and function.

CONCLUSIONS: Patients with OA of the knee exhibit lower isokinetic strength in the hip muscles than healthy control subjects. Strengthening the muscles surrounding the hip joint may help to decrease pain in people with knee OA. Some correlations between pain/function and peak torque were found.

KEYWORDS: Hip muscles; Isokinetic assessment; Strength; Peak torque; Knee osteoarthritis.

Costa RA, de Oliveira LM, Watanabe SH, Jones A, Natour J. Isokinetic assessment of the hip muscles in patients with osteoarthritis of the knee. Clinics. 2010;65(12):1253-1259.

Received for publication on July 23, 2010; First review completed on August 4, 2010; Accepted for publication on September 8, 2010

E-mail: jnatour@unifesp.br

Tel.: 5511 5576-4239

\section{INTRODUCTION}

Osteoarthritis (OA) is one of the most common joint diseases and the knee is the most frequently affected weight-bearing joint. ${ }^{1-8} \mathrm{OA}$ of the knee can cause functional disabilities-reduced lower limb muscle strength is marked in people with symptomatic knee OA-and several studies have suggested that knee extensor and knee flexor strength are both lost with established symptomatic disease. ${ }^{9,10} \mathrm{~A}$ variety of exercise programs for the treatment of knee OA have been described in the literature, which focus on improving the quadriceps strength. ${ }^{11,12}$ However, little attention has been paid to other lower limb muscle groups such as the hip muscles. ${ }^{13}$ Recent evidence suggests that the strength of hip abductor and adductor muscles may be important for reducing knee adduction moment, which is normally increased in patients with knee OA and which is related to symptom severity and disease progression. ${ }^{14}$

Yamada et al. ${ }^{15}$ found that patients with knee OA had stronger hip adductors than aged-matched controls, and

Copyright (c) 2010 CLINICS - This is an Open Access article distributed under the terms of the Creative Commons Attribution Non-Commercial License (http:// creativecommons.org/licenses/by-nc/3.0/) which permits unrestricted noncommercial use, distribution, and reproduction in any medium, provided the original work is properly cited. those with severe OA had even stronger adductors. However, measurement in that study was carried out isometrically.

The isokinetic test can provide data about maximal strength throughout the range of motion (ROM) as the muscle contracts, allowing the quantification of parameters such as strength, work and resistance. ${ }^{16-21}$ No study reporting the isokinetic strength of the hip muscle in patients with knee OA was found in the literature

Our study aimed first, to evaluate the difference in isokinetic assessment of hip muscles between patients with knee OA and matched healthy controls, and second, to evaluate the correlation between the isokinetic strength of hip muscles and pain and function in patients with knee OA.

\section{MATERIALS AND METHODS}

This study was approved by the ethics committee of the Federal University of Sao Paulo. Patients who fulfilled the inclusion criteria and agreed to participate signed an informed consent form and were included in the study. One hundred subjects were studied: 50 patients with a diagnosis of OA of the knee (25 with unilateral knee OA and 25 with bilateral knee OA) and 50 controls. The patients were recruited from the OA clinics and the controls from the university community. Diagnosis of knee OA was made by 
a rheumatologist. Inclusion criteria for the patients were diagnosis of knee OA using the clinical and radiographic criteria of the American College of Rheumatology; ${ }^{22}$ visual analog scale (VAS) for knee pain ranging from 2 to $8 \mathrm{~cm}$ on a $10 \mathrm{~cm}$ scale; and an algofunctional index (Lequesne) ranging from 5 to 12 . The control group was matched by age, gender and body mass index (BMI).

Subjects aged $<18$ and $>65$ years; patients with any type of other lower limb disease; knee and/or hip prosthesis; joint instabilities and having undergone lower limb surgery; patients with a heart condition; patients with uncontrolled hypertension; patients with coagulopathy undergoing anticoagulant therapy; pregnant women; patients with fibromyalgia; and individuals unable to perform the test were excluded from both groups.

Before the isokinetic muscle test, all subjects were evaluated by an assessor blinded to group allocation using:

- An assessment chart containing data on personal characteristics; measurement of weight and height to calculate the BMI; radiologic evaluation of the knees according to the criteria of Kellgren and Lawrence $(1957)^{23}$ for OA of the knee; knee deformity (radiographic analysis - the knee was defined as varus when alignment was more than $0^{\circ}$ in the varus direction, valgus when it was more than $0^{\circ}$ in the valgus direction, and neutral when alignment was 0 ); goniometry of the hip and knees; and lower limb dominance (preference for kicking);

- VAS for knee pain in the previous week using a $10 \mathrm{~cm}$ scale, where 0 means no pain and 10 means worse pain; $^{24}$

- The Lequesne Index for OA of the knee, which evaluates pain/discomfort, maximum distance walked and activities of daily living. Scores range from 0 to 24 , with higher scores indicating greater disease severity; ${ }^{25}$

- The Western Ontario and McMaster Universities OA Index (WOMAC), which consists of three subscales: pain, stiffness and physical function. Scores range from 0 to 96, with higher scores indicating greater disease severity. $^{26}$

Computerized isokinetic assessments of hip muscles were performed by another blinded assessor on a Cybex isokinetic dynamometer (Norm model, Cybex International, Inc, Ronkonkoma, NY, USA). Before the isokinetic test, all subjects warmed up on a stationary bicycle (Metabolic System Bike, Cybex - Division of Lumex) at a mean speed of $60 \mathrm{rpm}$ for $5 \mathrm{~min}$. All individuals were told how to carry out the test correctly and repeated each movement three times at the velocities to be tested in order to become familiar with the apparatus.

The recommendations for the position and angular velocities suggested by the manufacturer of the apparatus (Cybex International, Inc, 1997) ${ }^{27}$ were followed for all movements tested owing to controversies in the literature about angular velocities. The isokinetic test protocol followed the recommendations of Perrin (1993). ${ }^{20}$ Figure 1 shows the positions used with Cybex for the evaluations. The movements used during the test were flexion-extension at 30,60 and $180 \%$ s, abduction-adduction at 30, 120 and $240 \% \mathrm{~s}$ and medial-lateral rotation at 30 and $60 \%$ s. Slower velocities were used to evaluate strength, middle velocities

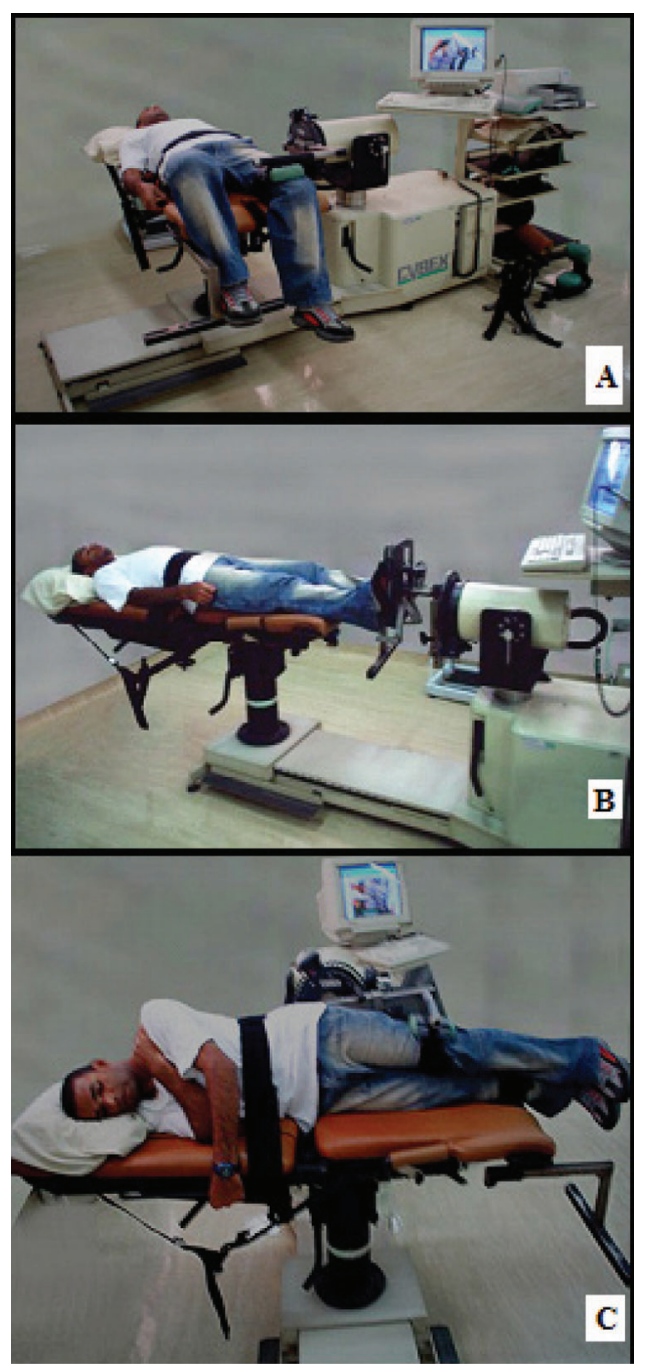

Figure 1 - Position for performing the flexion-extension movements $(A)$, abduction-adduction movements $(B)$, and medial and lateral rotation movements (C).

to evaluate power, and the faster velocities to evaluate resistance. $^{20}$

The sequence of movements was randomized to prevent the influence of fatigue on performance. The apparatus was calibrated before the test, compensation was made for the action of gravity during flexion-extension and abductionadduction movements and a correction for weight was made for each subject in all evaluations.

All individuals were told how to carry out the test correctly and repeated each movement three times at the velocities to be tested in order to become familiar with the apparatus. During these three repetitions the ROM was adjusted individually for each patient. Then the movements were repeated five times at each velocity and the mean of these five attempts was used as the final score. There was always a $30 \mathrm{~s}$ resting period between each attempt and between each change in angular velocity. Additionally, subjects received verbal encouragement so that they would apply the maximal force possible

The data were analyzed using the SPSS version 10.0. The following tests were used: Mann-Whitney test, Pearson's $\chi^{2}$ test and $t$ test to compare the groups' homogeneity; the 
Table 1 - Demographic characteristics and goniometry values of patients with osteoarthritis (OA) of the knee and control subjects.

\begin{tabular}{|c|c|c|c|}
\hline & OA group $(n=50)$ & Control group $(n=50)$ & p Value \\
\hline Gender (female/male) & $44 / 6$ & $44 / 6$ & 1 \\
\hline Age (years)* & $56(30-63)$ & $57(34-65)$ & 0.147 \\
\hline BMI $\left(\mathrm{kg} / \mathrm{m}^{2}\right)$ * & $29.09(16.92-41.33)$ & $28.17(17.22-35.49)$ & 0.082 \\
\hline Knee deformity (varus/valgus) & $39 / 11$ & $33 / 17$ & 0.097 \\
\hline Lower limb dominance (R/L) & $27 / 23$ & $42 / 8$ & 0.181 \\
\hline Knee flexion R (degrees) & $96(82-100)$ & $120(95-125)$ & $<0.001 *$ \\
\hline Knee flexion L (degrees) & $92(90-100)$ & $119(95-125)$ & $<0.001$ * \\
\hline Knee extension $\mathrm{R}$ (degrees) & $2.5(0-5)$ & 0 & 0.781 \\
\hline Knee extension L (degrees) & 0 & 0 & 1 \\
\hline Hip flexion R (degrees) & $89(67-105)$ & $100(90-125)$ & $<0.001$ * \\
\hline Hip flexion L (degrees) & $87(76-102)$ & $102(90-123)$ & $<0.001$ * \\
\hline Hip extension R (degrees) & $8(4-10)$ & $9(7-10)$ & 0.835 \\
\hline Hip extension L (degrees) & $7(4-9)$ & $9(7-10)$ & 0.623 \\
\hline Hip adduction R (degrees) & $9(8-13)$ & $9(6-15)$ & 0.789 \\
\hline Hip adduction L (degrees) & $9(7-10)$ & $9(7-15)$ & 0.912 \\
\hline Hip abduction R (degrees) & $36(24-43)$ & $40(33-45)$ & 0.345 \\
\hline Hip abduction L (degrees) & $34(21-40)$ & $40(33-44)$ & 0.428 \\
\hline Hip medial rotation $\mathrm{R}$ (degrees) & $32(19-42)$ & $40(33-45)$ & 0.278 \\
\hline Hip medial rotation L (degrees) & $31(19-37)$ & $40(33-45)$ & 0.134 \\
\hline Hip lateral rotation $\mathrm{R}$ (degrees) & $32(15-40)$ & $41(39-45)$ & 0.278 \\
\hline Hip lateral rotation L (degrees) & $31(15-38)$ & $40(36-44)$ & 0.189 \\
\hline
\end{tabular}

*Median (range); $\mathrm{BMI}=$ body mass index; $\mathrm{R}=$ right; $\mathrm{L}=$ left; $\mathrm{p}=\mathrm{p}$ value between groups.

two-way analysis of variance test with a Bonferroni correction to compare the OA groups and the control group for peak torque; and Pearson's and Spearman's correlation coefficient to find the correlation of the peak torque with VAS for pain, Lequesne and WOMAC scores. The level of significance was set at 0.05 or $5 \%$ for all tests.

\section{RESULTS}

The control group and the group of patients with knee OA were matched for gender, age, BMI, knee deformity and lower limb dominance (Table 1). Of the 50 patients with OA, $25(50 \%)$ had unilateral knee OA (11 $(22 \%)$ had OA in the right (R) knee and $14(28 \%)$ had OA in the left (L) knee) and $25(50 \%)$ had bilateral knee OA. Median duration of the disease was 8 years among the patients with OA of the knee (7.5 in the unilateral OA group and 10 years in the bilateral OA group). Both groups were assessed for goniometry of the knees and hips and statistically significant differences were found for knee and hip flexion (Table 1).

Table 2 shows the scores of the patients on the VAS for pain, the Lequesne index for the knee and hip and the WOMAC index.

To facilitate the understanding the results were divided into two groups the group with unilateral OA and the group with bilateral OA.

Table 2 - VAS scores for knee, Lequesne scores and WOMAC scores of patients with OA of the knee.

\begin{tabular}{lcc}
\hline & $\begin{array}{c}\text { Unilateral OA } \\
\text { group }(\mathbf{n}=25)\end{array}$ & $\begin{array}{c}\text { Bilateral OA } \\
\text { group }(\mathbf{n}=\mathbf{2 5})\end{array}$ \\
\hline VAS & $6(3-7)$ & $5(3-8)$ \\
Knee Lequesne & $8(4-12)$ & $10(7-12)$ \\
WOMAC & $51(17-92)$ & $80(52-92)$ \\
\hline
\end{tabular}

Data are reported as median (range).

OA, osteoarthritis; VAS, visual analog scale; WOMAC, Western Ontario and McMaster Universities Osteoarthritis Index.
Isokinetic Dynamometry - Peak Torque (PT)

Unilateral OA Group. We found a significantly lower peak torque $(\mathrm{p}<0.001)$ for the contralateral hip in comparison with the control group for the flexion, extension, abduction, adduction, medial rotation and lateral rotation at all velocities. Peak torque values for the ipsilateral hip were significantly lower in the OA group than for the control group for flexion, extension, medial rotation and lateral rotation at all velocities and for abduction at $120 \% \mathrm{~s}$ and adduction at $30 \% \mathrm{~s}(\mathrm{p}<0.001)$ (Table 3$)$.

Bilateral OA Group. We found a significantly lower peak torque $(\mathrm{p}<0.001)$ for both hips in the bilateral OA group than for the control group for the flexion, extension, medial rotation and lateral rotation at all velocities. Peak torque values for both the $\mathrm{R}$ and $\mathrm{L}$ hip were significantly lower in the OA group than for the control group for hip abduction at $30 \% \mathrm{~s}$ and $120 \% \mathrm{~s}(\mathrm{p}<0.001)$ and hip adduction at $30 \% \mathrm{~s}$ and $120 \%$ s $(\mathrm{p}<0.001)$ (Table 3$)$.

\section{Correlations}

Unilateral OA group. Correlating peak torque of the hip with VAS for pain, we found a strong correlation in the ipsilateral hip for extension at $180 \%$ s, adduction at $240 \% \mathrm{~s}$, and medial rotation at $30 \%$ s. In the correlation between peak torque and the Lequesne score, we found strong correlations in the contralateral hip for extension at $30 \%$, $60 \%$, and $180 \% \mathrm{~s}$ and medial rotation at $60 \%$ s. In the correlation between peak torque and WOMAC score, we found a strong correlation in the contralateral hip for extension at $30 \%, 60 \%$, and $180 \%$ and in the ipsilateral hip for abduction at $30 \%$ and $120 \% \mathrm{~s}$ and adduction at $120 \%$ s (Table 4). There was no correlation between Kellgren and Lawrence degree and peak torque.

Bilateral OA Group. Correlating the peak torque and VAS for pain, we observed a high correlation for the right hip in lateral rotation at $30 \%$ s. We also found a strong correlation between peak torque and Lequesne score, in the right hip for extension at $30 \% \mathrm{~s}$ and $60 \%$, adduction at 
Table 3 - Peak torque values $(\mathrm{Nm})$ of the in patients with OA of the knee and controls.

\begin{tabular}{|c|c|c|c|c|c|c|}
\hline & $\begin{array}{l}\text { Unilateral OA } \\
\text { group }(n=25)\end{array}$ & $\begin{array}{l}\text { Unilateral Control } \\
\text { group }(n=25)\end{array}$ & p Value & $\begin{array}{l}\text { Unilateral OA } \\
\text { group }(n=25)\end{array}$ & $\begin{array}{l}\text { Unilateral Control } \\
\text { group }(n=25)\end{array}$ & p Value \\
\hline & \multicolumn{3}{|c|}{ Contralateral hip } & \multicolumn{3}{|c|}{ Ipsilateral hip } \\
\hline Flex $30 \% \mathrm{~s}$ & $96.72(25.47)$ & $127.20(57.41)$ & $0.019 *$ & $98.92(29.38)$ & 127.04 (57.35) & 0.034 * \\
\hline Flex $60 \% \mathrm{~s}$ & $82.40(22.93)$ & $112.56(52.16)$ & $0.011 *$ & $85.84(33.87)$ & 111.48 (49.54) & $0.038^{*}$ \\
\hline Flex $180 \%$ s & $50.76(13.40)$ & $74.00(44.83)$ & $0.017 *$ & $52.20(15.94)$ & 70.28 (37.92) & $0.033^{*}$ \\
\hline Ext $30 \% \mathrm{~s}$ & 90.72 (38.29) & $170.92(86.46)$ & $<0.001 *$ & 93.92 (43.73) & 166.68 (76.47) & $<0.001$ * \\
\hline Ext $60 \% \mathrm{~s}$ & 79.32 (35.63) & 167.52 (73.34) & $<0.001 *$ & $90.76(46.91)$ & 155.28 (71.19) & $<0.001$ * \\
\hline Ext $180 \%$ s & $24.28(21.08)$ & $112.40(82.03)$ & $<0.001 *$ & $15.80(28.48)$ & $87.56(65.94)$ & $<0.001$ * \\
\hline $\mathrm{Abd} 30 \% \mathrm{~s}$ & $58.76(17.74)$ & $82.04(29.25)$ & $0.001 *$ & $68.44(26.54)$ & $79.84(28.64)$ & 0.151 \\
\hline Abd $120 \% \mathrm{~s}$ & $40.48(15.58)$ & $61.48(28.63)$ & $0.002 *$ & $44.32(21.37)$ & $59.84(27.49)$ & 0.031 * \\
\hline $\mathrm{Abd} 240 \% \mathrm{~s}$ & $31.20(9.21)$ & $37.60(11.40)$ & $0.034 *$ & 35.20 (11.96) & $34.44(13.64)$ & 0.835 \\
\hline Add $30 \% \mathrm{~s}$ & $43.72(19.11)$ & 83.84 (37.89) & $<0.001 *$ & $36.32(30.04)$ & $72.80(36.78)$ & $<0.001$ * \\
\hline Add $120 \% \mathrm{~s}$ & $18.40(16.59)$ & $44.36(39.80)$ & $0.004 *$ & $29.88(44.08)$ & $40.12(34.15)$ & 0.363 \\
\hline Add $240 \% \mathrm{~s}$ & $11.56(6.42)$ & $11.24(20.42)$ & $0.028^{*}$ & $14.28(29.65)$ & 8.64 (14.79) & 0.399 \\
\hline $\mathrm{Rm} \mathrm{30 \% \textrm {s }}$ & $13.00(5.74)$ & $19.28(8.44)$ & $0.003 *$ & $11.50(5.75)$ & $18.40(8.90)$ & $0.002 *$ \\
\hline $\mathrm{Rm} 60 \% \mathrm{~s}$ & $11.04(5.37)$ & $18.32(9.68)$ & $0.002^{*}$ & $10.44(7.04)$ & $17.20(10.41)$ & 0.010 * \\
\hline $\mathrm{RI} 30 \% \mathrm{~s}$ & 16.40 (7.95) & $24.04(14.64)$ & $0.026^{*}$ & $13.24(7.26)$ & $25.16(14.80)$ & 0.001 * \\
\hline \multirow[t]{3}{*}{$\mathrm{RI} 60 \% \mathrm{~s}$} & $13.52(5.47)$ & $21.00(13.77)$ & $0.015^{*}$ & $11.40(5.28)$ & $22.04(13.14)$ & $<0.001 *$ \\
\hline & $\begin{array}{c}\text { Bilateral OA } \\
\text { group }(n=25)\end{array}$ & $\begin{array}{l}\text { Bilateral Control } \\
\text { group }(n=25)\end{array}$ & p Value & $\begin{array}{c}\text { Bilateral OA } \\
\text { group }(n=25)\end{array}$ & $\begin{array}{l}\text { Bilateral Control } \\
\text { group }(n=25)\end{array}$ & p Value \\
\hline & \multicolumn{3}{|c|}{ Right hip } & \multicolumn{3}{|c|}{ Left hip } \\
\hline Flex $30 \% \mathrm{~s}$ & $72.64(33.12)$ & $144.12(50.97)$ & $<0.001 *$ & 77.16 (34.68) & $142.52(56.78)$ & $<0.001$ * \\
\hline Flex $60 \% \mathrm{~s}$ & $61.20(30.57)$ & $121.84(43.72)$ & $<0.001 *$ & $64.68(32.84)$ & $122.44(48.50)$ & $<0.001$ * \\
\hline Flex $180 \%$ s & $39.16(18.16)$ & 79.04 (32.45) & $<0.001 *$ & $40.52(18.71)$ & $82.16(41.43)$ & $<0.001$ * \\
\hline Ext $30 \%$ & $66.36(50.67)$ & $192.84(57.75)$ & $<0.001 *$ & $80.44(47.07)$ & $177.12(58.22)$ & $<0.001$ * \\
\hline Ext $60 \% \mathrm{~s}$ & $57.28(47.98)$ & $174.36(45.36)$ & $<0.001 *$ & $59.52(50.44)$ & 163.84 (53.29) & $<0.001$ * \\
\hline Ext $180 \%$ s & $18.40(23.58)$ & $105.76(47.24)$ & $<0.001 *$ & 17.16 (23.99) & $108.48(72.05)$ & $<0.001$ * \\
\hline Abd $30 \% \mathrm{~s}$ & $53.68(27.45)$ & 78.40 (32.29) & $0.005^{*}$ & $54.92(24.94)$ & $84.32(30.20)$ & $<0.001$ * \\
\hline Abd $120 \% / s$ & $33.80(16.68)$ & $57.04(27.32)$ & $0.001 *$ & 34.52 (16.91) & $61.28(25.85)$ & $<0.001$ * \\
\hline Abd $240 \% / s$ & $30.64(14.70)$ & $32.80(10.21)$ & 0.549 & $28.92(13.82)$ & $36.24(12.48)$ & 0.055 \\
\hline Add $30 \% \mathrm{~s}$ & $32.20(20.08)$ & $69.56(24.63)$ & $<0.001 *$ & $40.64(18.66)$ & $68.52(21.30)$ & $<0.001$ * \\
\hline Add $120 \% \mathrm{~s}$ & $10.16(13.06)$ & $33.36(15.02)$ & $<0.001 *$ & 9.96 (12.93) & 30.32 (19.65) & $<0.001$ * \\
\hline Add $240 \% \mathrm{~s}$ & $4.8(10.73)$ & $5.2(11.77)$ & 0.881 & $4.48(10.61)$ & $4.12(10.37)$ & 0.904 \\
\hline $\mathrm{Rm} \mathrm{30 \% \textrm {s }}$ & $12.28(5.89)$ & $19.36(6.28)$ & $<0.001 *$ & $9.12(4.42)$ & $19.44(7.01)$ & $<0.001$ * \\
\hline $\mathrm{Rm} 60 \% \mathrm{~s}$ & $10.56(4.74)$ & $17.88(6.51)$ & $<0.001 *$ & $8.76(4.91)$ & $19.16(8.70)$ & $<0.001$ * \\
\hline $\mathrm{RI} 30 \% \mathrm{~s}$ & $11.64(5.31)$ & $23.84(8.36)$ & $<0.001$ * & $13.40(4.07)$ & 24.72 (12.94) & $<0.001$ * \\
\hline $\mathrm{RI} 60 \% \mathrm{~s}$ & 9.76 (3.93) & $21.36(7.23)$ & $<0.001 *$ & $13.40(7.10)$ & $22.24(10.19)$ & 0.001 * \\
\hline
\end{tabular}

Data are reported as median (rage); Flex = flexion; Ext = extension; Abd = abduction; Add = adduction; $\mathrm{Rm}=\mathrm{Medial}$ rotation; $\mathrm{RI}=$ lateral rotation; $\mathrm{p}$ $=p$ value between groups;

*significant $p$ value $(p<0.05)$.

$30 \%$, medial rotation at $30 \% \mathrm{~s}$ and lateral rotation $30 \% \mathrm{~s}$, and in the left hip for extension at $30 \%$ s, medial rotation at $30 \%$ and $60 \%$ and lateral rotation at $60 \%$ s. There was a strong correlation between peak torque and the WOMAC score in the right and the left hip for adduction at $240 \%$ s (Table 4 ). There was no correlation between Kellgren and Lawrence degree and peak torque.

\section{DISCUSSION}

As far as we know, this is the first study to evaluate the isokinetic hip strength in patients with knee OA. We show that these patients have lower peak torque in hip muscles for all movements in comparison with matched controls. The patients with unilateral or bilateral knee OA had similar results, indicating that hip muscles on both sides are weak in patients with knee OA whether the disease is unilateral or bilateral. A possible explanation for this finding is that owing to the joint pain, patients with OA have changes in muscle function and biomechanics.

Our results appeared to show that patients with bilateral OA had weaker muscles than patients with unilateral OA, but this cannot be confirmed as this comparison was not the aim of our study.

In this study, the groups were matched for gender and age and there was a predominance of women, which is similar to the findings reported in other studies on the prevalence of the disease. $4,5,7,17,18,21,28,29$

The lack of correlation between Kellgren and Lawrence degree and isokinetic strength was unexpected and suggests that the radiological findings are not related to strength, power or resistance as evaluated by Cybex.

No significant difference was seen between the two groups in the type of knee deformity (varus and valgus), and there was a predominance of varism, which is in agreement with the literature. Some authors have demonstrated that in patients with medial compartmental OA of the knee, the hip adductor muscles may become strengthened to decrease varus deformity of the limb ${ }^{15}$; this was not found in our patients and controls despite their varism predominance.

Afzali et al. ${ }^{30}$ found no relationship between quadriceps strength and the dominant leg for individuals with knee diseases. Toyonaga et al. ${ }^{31}$ observed no difference in peak 
Table 4 - Correlation coefficient between peak torque of the hip and visual analogue scale for pain, Lequesne and WOMAC scores in patients with OA of the knee.

\begin{tabular}{|c|c|c|c|c|c|c|}
\hline & \multicolumn{6}{|c|}{ Unilateral OA group $(n=25)$} \\
\hline & \multicolumn{3}{|c|}{ Contralateral hip } & \multicolumn{3}{|c|}{ Ipsilateral hip } \\
\hline & VAS & Lequesne & WOMAC & VAS & Lequesne & WOMAC \\
\hline Flex $30 \%$ & 0.381 & -0.072 & 0.078 & -0.059 & 0.009 & 0.154 \\
\hline Flex $60 \% / \mathrm{s}$ & -0.075 & -0.251 & -0.162 & 0.055 & $-0.415^{*}$ & 0.225 \\
\hline Flex $180 \%$ & -0.316 & -0.073 & -0.145 & -0.074 & -0.342 & 0.165 \\
\hline Ext $30 \%$ & -0.003 & $-0.551 *$ & $-0.415^{*}$ & -0.025 & -0.376 & 0.232 \\
\hline Ext $60 \%$ & 0.196 & $-0.566^{*}$ & $-0.437^{*}$ & -0.081 & $-0.528^{*}$ & -0.573 \\
\hline Ext $180 \% \mathrm{~s}$ & -0.285 & $-0.430 *$ & $-0.482 *$ & $-0.434^{\star}$ & -0.041 & 0.285 \\
\hline Abd $30 \%$ & 0.038 & 0.034 & -0.153 & 0.247 & $-0.207^{*}$ & $0.423 *$ \\
\hline Abd $120 \%$ & 0.113 & -0.258 & -0.143 & 0.221 & $-0.231 *$ & 0.178 \\
\hline Abd $240 \%$ & 0.174 & -0.089 & -0.327 & 0.269 & -0.134 & $0.387 *$ \\
\hline Add $30 \% \mathrm{~s}$ & 0.373 & -0.206 & 0.212 & -0.046 & -0.004 & 0.026 \\
\hline Add $120 \% \mathrm{~s}$ & 0.367 & -0.392 & -0.345 & 0.228 & $0.399 *$ & $-0.382^{*}$ \\
\hline Add $240 \% \mathrm{~s}$ & 0.193 & 0.038 & -0.189 & $0.398^{*}$ & -0.003 & -0.163 \\
\hline $\mathrm{Rm} \mathrm{30} / \mathrm{s}$ & 0.168 & -0.121 & -0.104 & 0.021 & -0.260 & 0.065 \\
\hline $\mathrm{Rm} 60 \% \mathrm{~s}$ & -0.111 & $-0.548^{*}$ & -0.387 & -0.174 & -0.214 & 0.346 \\
\hline $\mathrm{RI} 30 \% \mathrm{~s}$ & -0.147 & 0.160 & -0.369 & $-0.556^{\star}$ & -0.280 & -0.084 \\
\hline \multirow[t]{4}{*}{$\mathrm{RI} 60 \% \mathrm{~s}$} & 0.075 & -0.039 & -0.352 & -0.324 & -0.309 & -0.273 \\
\hline & \multicolumn{6}{|c|}{ Bilateral OA group $(n=25)$} \\
\hline & \multicolumn{3}{|c|}{ Right hip } & \multicolumn{3}{|c|}{ Left hip } \\
\hline & VAS & Lequesne & WOMAC & VAS & Lequesne & WOMAC \\
\hline Flex $30 \%$ & -0.019 & -0.316 & -0.172 & 0.146 & 0.002 & -0.057 \\
\hline Flex $60 \% \mathrm{~s}$ & -0.062 & -0.380 & -0.163 & 0.124 & 0.020 & -0.066 \\
\hline Flex $180 \% / \mathrm{s}$ & 0.071 & -0.271 & -0.149 & 0.295 & 0.181 & -0.095 \\
\hline Ext $30 \%$ & -0.084 & $-0.524 *$ & 0.237 & 0.128 & $0.418^{*}$ & 0.307 \\
\hline Ext $60 \% \mathrm{~s}$ & 0.006 & $-0.388^{*}$ & 0.217 & 0.004 & -0.367 & 0.193 \\
\hline Ext $180 \% \mathrm{~s}$ & 0.223 & -0.212 & 0.126 & 0.004 & 0.004 & 0.261 \\
\hline Abd $30 \% \mathrm{~s}$ & 0.305 & 0.048 & -0.003 & 0.214 & -0.64 & -0.177 \\
\hline Abd $120 \% \mathrm{~s}$ & 0.151 & -0.158 & -0.131 & 0.099 & 0.060 & -0.253 \\
\hline Abd $240 \% \mathrm{~s}$ & 0.179 & -0.034 & -0.191 & 0.226 & 0.135 & -0.337 \\
\hline Add $30 \% \mathrm{~s}$ & 0.249 & $-0.549 *$ & 0.247 & 0.070 & -0.184 & -0.072 \\
\hline Add $120 \% \mathrm{~s}$ & -0.008 & -0.276 & 0.258 & -0.102 & -0.109 & 0.204 \\
\hline Add $240 \% \mathrm{~s}$ & 0.093 & -0.142 & $0.408^{*}$ & 0.208 & 0.099 & $0.402 *$ \\
\hline $\mathrm{Rm} \mathrm{30} / \mathrm{s}$ & 0.158 & $0.442 *$ & 0.01 & -0.131 & $0.438 *$ & 0.144 \\
\hline $\mathrm{Rm} 60 \% \mathrm{~s}$ & 0.023 & 0.350 & -0.093 & -0.211 & $-0.474 *$ & -0.167 \\
\hline $\mathrm{RI} 30 \% \mathrm{~s}$ & $0.415^{*}$ & $0.433^{*}$ & -0.054 & 0.238 & 0.144 & 0.1 \\
\hline $\mathrm{RI} 60 \% \mathrm{~s}$ & -0.063 & 0.361 & -0.091 & -0.167 & $0.484 *$ & -0.083 \\
\hline
\end{tabular}

Data are reported as correlation coefficient ( $\rho$ ); PT Flex = flexion peak torque; PT Ext = extension peak torque; PT Abd = abduction peak torque; PT Add = adduction peak torque; PT Rm = Medial rotation peak torque; PT RI = lateral rotation peak torque; VAS = visual analog scale for pain;

*significant correlation $(\mathrm{p}<0.05)$.

torque between the dominant and non-dominant leg in healthy men or women. For the hip rotator musculature, studies have found no differences in muscle strength that could be related to lower limb dominance. In our study, most patients in the control group and half of the patients with knee OA exhibited dominance of the right lower limb.

Goniometry of the knee revealed limited ROM of the affected knee for both flexion and extension in all patients with OA in comparison with the control group. For hip ROM, a significant difference was also seen between patients with unilateral or bilateral $\mathrm{OA}$ and the control group. Goniometry of the hip obtained for these patients revealed limited ROM for almost all movements assessed, thereby demonstrating loss of hip ROM in patients with $\mathrm{OA}$ of the knee. Despite the biarticular musculature of both joints, no reports correlating limited knee ROM with hip ROM are available in the literature. In the present study, limited ROM was also observed for the contralateral hip.
There is a lack of studies investigating the relationship between knee diseases and their effect on the hip muscles. Similar studies correlating other muscles of the lower limbs have been described. Lane and Buckwalter ${ }^{5}$ correlated the gastrocnemius and anterior tibial muscles with peak torque of the ankle and knee and demonstrated that peak torque of the knee flexors and ankle extensors is altered by the action of the gastrocnemius and anterior tibial muscles. McCarthy et al. ${ }^{32}$ submitted healthy women over 60 years of age to hip, knee and ankle isokinetic exercises and demonstrated a correlation between the peak torque of the hip flexors and extensors, knee flexors and extensors, and ankle plantar and dorsal flexors at $60 \%$ s. In our study, significant differences in the peak torque of the flexor/extensor, abductor/adductor, and medial/lateral hip rotator muscles were observed between patients with unilateral or bilateral OA of the knee and controls.

In contrast to the results of isokinetic tests on the knee and ankle reported in the literature, few studies have documented 
alterations in the peak torque of hip muscles, especially for abductors and adductors in patients with hip OA. Arokoski et al. (2002) $)^{33}$ analyzed hip muscle strength in patients with OA of the hip and observed a strong correlation between peak torque and hip pain, as rated on a VAS, probably owing to the joint involvement of the disease and hip muscle weakness in patients with OA. Comparisons of peak torque of the hip between individuals with and without OA of the hip revealed a lower peak torque for hip flexion and extension movements at velocities of 60 and $120 \%$ s for patients with knee OA in comparison with controls. The flexor muscles were weaker and no significant difference between groups was observed during extension, demonstrating that the affected side was weaker than the non-affected side and that men with OA of the hip have lower flexion strength than controls. Peak torque values obtained for hip abduction and adduction movements were also lower than in controls, indicating lower hip abductor and adductor muscle strength in patients with OA of the hip. No studies analyzing isokinetic dynamometry values of the hip in patients with OA of the knee are available in the literature. We found only reports of isokinetic assessments of the hip in healthy individuals and patients with OA of the hip. ${ }^{31,32,34-36}$

Callaghan et al. $(2000)^{37}$ evaluated hip muscle strength in healthy individuals and demonstrated that the extensor muscles are the strongest muscle group, followed by the flexor, adductor and abductor muscles and, finally, the rotator muscles. Moreover, the medial rotator muscles were stronger than the lateral rotator muscles. Likewise, comparisons of muscle strength between younger and older individuals revealed $60 \%$ higher peak torque values in the former group. In our study in the control group the extensor muscles are the strongest muscle group, findings that are similar to those of Callaghan et al. ${ }^{37}$ However, in the OA groups the flexor muscles are the strongest muscle group and this must be taken into consideration when rehabilitating these patients.

Burnfield et al. ${ }^{38}$ determined the influence of isokinetic peak torque values of the hip and knee flexor and extensor muscles and the ankle plantar and dorsal flexor muscles at a velocity of $60 \%$ s. Peak torque was higher for the hip extensors and knee flexors, while the lowest values were observed for the plantar and dorsal flexors of the ankle.

Pohtilla et al. ${ }^{39}$ found that individuals with weak hip extensor muscles assume a characteristic position of the pelvis in relation to the femur when in an erect position, which may result in postural alterations leading to changes in the lower limb musculature. This suggests that most of our patients with limited ROM in the knee and/or hip could exhibit postural alterations such as knee flexure, altering the ROM of the hip owing to the biarticular musculatures of the two joints. These postural alterations need to be assessed individually during the treatment of these patients, and, when necessary, work on posture could be added to the rehabilitation protocol.

Our results show that patients with knee OA have weaker hip muscles bilaterally than healthy controls. So exercise for strengthening the hip muscles should be included in the rehabilitation program for patients with knee OA. Strengthening the muscles surrounding the hip joint would appear to have the potential to help decrease pain in people with knee OA.

The correlations found between the peak torque and function (Lequesne and WOMAC) were stronger than the correlation with pain. This might suggest that exercise to strengthen hip muscles would improve this function. Future studies are necessary to answer this question.

One limitation of our study was the small sample. Another limitation was that our sample had median impairment in function measured by the Lequesne index so we our results cannot be extrapolated for patients with better or worse functional level.

\section{CONCLUSIONS}

- Patients with OA of the knee exhibit lower isokinetic strength, power and resistance in the hip muscles than control subjects without OA of the knee.

- For function the unilateral OA group showed a correlation with PT of extensors and medial rotators in the contralateral hip and with PT of flexors, extensors, abductors and adductors in the ipsilateral hip. The bilateral OA group showed a correlation with PT of extensors, adductors, lateral rotators and medial rotators in the right hip and with PT of extensors, adductors, medial rotators and lateral rotators in the left hip.

- For function the unilateral OA group showed a correlation with PT of extensors and lateral rotators in the contralateral hip and with PT of abductors and adductors in the ipsilateral hip. The bilateral OA group showed a correlation with PT of extensors, adductors, lateral rotators and medial rotators in the right hip and with PT of extensors, adductors, medial rotators and lateral rotators in the left hip.

\section{REFERENCES}

1. Akeson WH, Garfin S, Amiel D, Woo SL. Para-articular connective tissue in osteoarthritis. Semin Arthritis Rheum. 1989;18(4 Suppl 2):41-50, doi: 10.1016/0049-0172(89)90015-2.

2. Dieppe P. Some recent clinical approaches to osteoarthritis research. Semin Arthritis Rheum. 1990;20(3 Suppl 1):2-11, doi: 10.1016/0049-0172 (90)90043-F.

3. Kovar PA, Allegrante JP, MacKenzie CR, Peterson MG, Gutin B, Charlson ME. Supervised fitness walking in patients with osteoarthritis of the knee. A randomized, controlled trial. Ann Intern Med. 1992;116: 529-34

4. Stelian J, Gil I, Habot B, Rosenthal M, Abramovici I, Kutok N, et al. Improvement of pain and disability in elderly patients with degenerative osteoarthritis of the knee treated with narrow-band light therapy. J Am Geriatr Soc. 2000;40:23-6.

5. Lane NE, Buckwalter JA. Exercise: a cause of osteoarthritis? Rheum Dis Clin North Am. 1993;19:617-33.

6. Ettinger WH Jr, Afable RF. Physical disability from knee osteoarthritis: the role of exercise as an intervention. Med Sci Sports Exerc. 1994; 26:1435-40

7. Zizic TM, Hoffman KC, Holt PA, Hungerford DS, O'Dell JR, Jacobs MA, et al. The treatment of osteoarthritis of the knee with pulsed electrical stimulation. J Rheumatol. 1995;22:1757-61.

8. Bland JH, Cooper SM. Osteoarthritis: a review of the cell biology involved and evidence for reversibility. Management rationally related to known genesis and pathophysiology. Semin Arthritis Rheum. 1984;14:106-33, doi: 10.1016/0049-0172(84)90002-7.

9. Hall KD, Hayes KW, Falconer J. Differential strength decline in patients with osteoarthritis of the knee: revision of a hypothesis. Arthritis Care Res. 1993;6:89-96, doi: 10.1002/art.1790060208.

10. Tan J, Balci N, Sepici V, Gener FA. Isokinetic and isometric strength in osteoarthrosis of the knee. A comparative study with healthy women. Am J Phys Med Rehabil. 1995;74:364-9, doi: 10.1097/00002060-19950900000008

11. Fransen M, Crosbie J, Edmonds J. Physical therapy is effective for patients with osteoarthritis of the knee: a randomized controlled clinical trial. J Rheumatol. 2001;28:156-64.

12. Huang $\mathrm{MH}$, Lin YS, Yang RC, Lee CL. A comparison of various therapeutic exercises on the functional status of patients with knee osteoarthritis. Semin Arthritis Rheum. 2003;32:398-406, doi: 10.1053/ sarh.2003.50021. 
13. Bennel KL, Hunt MA, Wrigjey TV, Hunter DJ, Hinman RS. The effects of hip muscle strengthening on knee load, pain, and function in people with knee osteoarthritis: a protocol for a randomised, single-blind controlled trial. BMC Musculoskelet Disord. 2007;8:121, doi: 10.1186/ 1471-2474-8-121.

14. Chang A, Hayes K, Dunlop D, Song J, Hurwitz D, Cahue S, et al. Hip abduction moment and protection against medial tibiofemoral osteoarthritis progression. Arthritis Rheum. 2005;52:3515-9, doi: 10.1002/art. 21406

15. Yamada H, Koshino T, Sakai N, Saito T. Hip adductor muscle strength in patients with varus deformed knee. Clin Orthop Relat Res. 2001;386:17985, doi: 10.1097/00003086-200105000-00023.

16. Fisher NM, Gresham G, Pendergast DR. Effects of a quantitative progressive rehabilitation program applied unilaterally to the osteoarthritic knee. Arch Phys Med Rehabil. 1993;74:1319-26, doi: 10.1016/00039993(93)90087-Q.

17. Fisher NM, White SC, Yack HJ, Smolinski RJ, Pendergast DR. Muscle function and gait in patients with knee osteoarthritis before and after muscle rehabilitation. Disabil Rehabil. 1997;19:47-55, doi: 10.3109/ 09638289709166827.

18. Cushnaghan J, McCarthy C, Dieppe P. Taping the patella medially: a new treatment for osteoarthritis of the knee joint? BMJ. 1994;308:753-5.

19. Malone TR. Concentric isokinetics. In: Timm KE. Orthopaedic physical therapy clinics of north America. Exercise Technologies: Philadelphia: W.B. Saunders Company; 1992:283-95.

20. Perrin DH. Terminology and the isokinetic torque curve. In: Perrin H (ed.). Isokinetic exercise and assesment. Champaign, USA, Human Kinetics Publishers; 1993:13-20.

21. Meireles SM, Oliveira LM, Andrade MS, Silva AC, Natour J. Isokinetic evaluation of the knee in patients with rheumatoid arthritis. Joint Bone Spine. 2002;69:566-73, doi: 10.1016/S1297-319X(02)00453-0.

22. Altman RD, Asch E, Bloch D, Bole G, Borestein D, Brandt K, et al. Development criteria for the classification and reporting of osteoarthritis. Classification of osteoarthritis of the knee. Diagnostic and Therapeutic Criteria Committee of the American Rheumatism Association. Arthritis Rheum 1986;29:1039-49, doi: 10.1002/art.1780290816.

23. Kellgren JH, Lawrence JS. Radiological assessment of osteo-arthrosis. Ann Rheum Dis. 1957;16:494-502, doi: 10.1136/ard.16.4.494.

24. Bellamy N. Osteoarthritis clinical trials: candidate variables and clinimetric properties. J Rheumatol. 1997;24:768-78.

25. Lequesne MG, Mery C, Samson M, Gerard P. Indexes of severity for osteoarthritis of the hip and knee. Validation-value in comparison with other assessment tests. Scand J Rheumatol Suppl. 1987;65:85-9, doi: 10. 3109/03009748709102182.

26. Bellamy N, Buchanan WW, Goldsmith $\mathrm{CH}$, Campbell J, Stitt LW. Validation study of WOMAC: a health status instrument for measuring clinically important patient relevant outcomes to antirheumatic drug therapy in patients with osteoarthritis of the hip or knee. J Rheumatol. 1988;15:1833-40.

27. Cybex Norm Int Cybex Norm INT-INC. Testing and rehabilitation system: pattern selection and set up: automated protocols. User's guide. Ronkonkoma, NY: Blue Sky Software Corporation; 1997.

28. Bagge E, Bjelle A, Edén S, Svanborg A. Osteoarthritis in the elderly: clinical and radiological findings in 79 and 85 year olds. Ann Rheum Dis. 1991;50:535-9, doi: 10.1136/ard.50.8.535.

29. Handy JR. Osteoarthritis in elderly knees. South Med J. 1996;89:1031-5, doi: 10.1097/00007611-199611000-00001.

30. Afzali L, Kuwabara F, Zachazewski J, Browne P, Robinson B. A new method for the determination of the characteristic shape of an isokinetic quadriceps femoris muscle torque curve. Phys Ther. 1992;72: 585-92.

31. Toyonaga T, Kawashima K. The measurement of muscle strength in the hip joint expressed by the octagonal diagram. Fukuoka Igaku Zasshi 1992;83:348-51.

32. McCarthy E, Horvat MA, Holtsberg PA, Wisenbaker JM. Repeated chair stands as a measure of lower limb strength in sexagenarian women. J Gerontol A Biol Sci Med Sci. 2004;59:1207-12.

33. Arokoski MH, Arokoski JPA, Haara M, Kankaanpää M, Vesterinen M, Niemitukia LH, et al. Hip muscle strength and muscle cross sectional area in men with and without hip osteoarthritis. J Rheumatol 2002;29: 2185-95

34. Helgeson K, Gajdosik RL. The stretch-shortening cycle of the quadriceps femoris muscle group measured by isokinetic dynamometry. J Orthop Sports Phys Ther. 1993;17:17-23.

35. Aagaard P, Simonsen EB, Trolle M, Bangsbo J, Klausen K. Isokinetic hamstring/quadriceps strength ratio influence from joint angular velocity, gravity correction and contraction mode. Acta Physiol Scand 1995;154:421-7, doi: 10.1111/j.1748-1716.1995.tb09927.x.

36. Calmels PM, Nellen M, van der Borne I, Jourdin P, Minaire P. Concentric and eccentric isokinet assessment of flexor-extensor torque ratios at the hip, knee, and ankle in a sample populations of healthy subjects. Arch Phys Med Rehabil. 1997;78:1224-30, doi: 10.1016/S0003-9993(97) 90336-1.

37. Callaghan JJ, Albright JC, Goetz DD, Olejniczak JP, Johnston RC. Charnley total hip arthroplasty with cement. Minimum twenty-five-year follow-up. J Bone Joint Surg Am. 2000;82:487-97.

38. Burnfield JM, Josephson KR, Powers CM, Rubenstein LZ. The influence of lower extremity joint torque on gait characteristics in elderly men. Arch Phys Med Rehabil. 2000;81:1153-7, doi: 10.1053/apmr.2000.7174.

39. Pohtilla JF. Kinesiology of hip extension at selected angles of pelvifemoral extension. Arch Phys Med Rehabil. 1969;50:241-50. 